\title{
Structure of the Main Protease from a Global Infectious Human Coronavirus, $\mathrm{HCoV}-\mathrm{HKU} 1^{\nabla} \dagger$
}

\author{
Qi Zhao, ${ }^{1}$ Shuang Li, ${ }^{1}$ Fei Xue, ${ }^{1}$ Yilong Zou, ${ }^{1}$ Cheng Chen, ${ }^{1}$ Mark Bartlam, ${ }^{2}$ and Zihe Rao ${ }^{1,2,3 *}$ \\ Tsinghua-Nankai-IBP Joint Research Group for Structural Biology, Tsinghua University, Beijing 100084, China ${ }^{1}$; College of \\ Life Sciences and Tianjin State Laboratory of Protein Sciences, Nankai University, Tianjin 300071, China ${ }^{2}$; and \\ National Laboratory of Biomacromolecules, Institute of Biophysics (IBP), Chinese Academy of Sciences, \\ Beijing 100101, China ${ }^{3}$
}

Received 11 February 2008/Accepted 10 June 2008

\begin{abstract}
The newly emergent human coronavirus HKU1 (HCoV-HKU1) was first identified in Hong Kong in 2005. Infection by HCoV-HKU1 occurs worldwide and causes syndromes such as the common cold, bronchitis, and pneumonia. The $\mathrm{CoV}$ main protease $\left(\mathrm{M}^{\mathrm{pro}}\right)$, which is a key enzyme in viral replication via the proteolytic processing of the replicase polyproteins, has been recognized as an attractive target for rational drug design. In this study, we report the structure of $\mathrm{HCoV}-\mathrm{HKU} 1 \mathrm{M}^{\mathrm{pro}}$ in complex with a Michael acceptor, inhibitor N3. The structure of $\mathrm{HCoV}-\mathrm{HKU} 1$ provides a high-quality model for group $2 \mathrm{~A} \mathrm{CoVs,} \mathrm{which} \mathrm{are} \mathrm{distinct} \mathrm{from} \mathrm{group}$ 2B CoVs such as severe acute respiratory syndrome $\mathrm{CoV}$. The structure, together with activity assays, supports the relative conservation at the $P 1$ position that was discovered by sequencing the HCoV-HKU1 genome. Combined with structural data from other $\mathrm{CoV} \mathrm{M}^{\mathrm{pro}} \mathrm{s}$, the $\mathrm{HCoV}-\mathrm{HKU} 1 \mathrm{M}^{\text {pro }}$ structure reported here provides insights into both substrate preference and the design of antivirals targeting CoVs.
\end{abstract}

Coronaviruses (CoVs) are positive-strand RNA viruses that have been identified as the main etiologic agents responsible for a vast number of enteric, gastric, and respiratory syndromes of both humans and animals (14-17, 19, 21, 23, 26, 30, 31, 34, 45). CoVs can be divided into three groups: group 1 (including human CoV 229E [HCoV 229E] and transmissible gastric enteritis virus [TGEV]), group 2 (including $\mathrm{HCoV}-\mathrm{OC} 43$, murine hepatitis virus [MHV], and bovine $\mathrm{CoV}$ [BCoV), and group 3 (including avian infectious bronchitis virus [IBV]). Shortly after the emergence of severe acute respiratory syndrome CoV (SARS-CoV) in 2003, group $2 \mathrm{CoVs}$ were further divided into two subgroups, termed $2 \mathrm{~A}$ and $2 \mathrm{~B}$ (46). The classical group 2 viruses constitute subgroup $2 \mathrm{~A}$, while the newly emergent SARS-CoV and its animal counterparts (37) form subgroup 2B. Group 1 and group $2 \mathrm{CoVs}$ have more impact on human health than group 3 , since group $3 \mathrm{CoVs}$ (such as avian IBV) can only infect avian species. Following the outbreak of SARS, group $2 \mathrm{CoVs}$ have continued to attract greater attention for two reasons. First, they consist of human viruses (SARS-CoV and $\mathrm{HCoV}-\mathrm{OC} 43$ ) as well as several important animal viruses ( $\mathrm{MHV}$ and $\mathrm{BCoV}$ ) that serve as useful models for CoV-host interactions. Second, group $2 \mathrm{CoVs}$ are reported to have crossed the animal-to-human species barrier in two instances: one bat-to-human transmission in group $2 \mathrm{~B}(27,37)$ and one transmission event in group $2 \mathrm{~A} \mathrm{CoVs}$, in which $\mathrm{BCoV}$ led to the emergence of $\mathrm{HCoV}-\mathrm{OC} 43$ (36).

Group 2A HCoVs were less widely studied prior to the global SARS epidemic in 2003. However, they are closely as-

\footnotetext{
* Corresponding author. Mailing address: Laboratory of Structural Biology, Life Sciences Building, Tsinghua University, Beijing 100084, China. Phone: 8610 62771493. Fax: 8610 62773145. E-mail: raozh @xtal.tsinghua.edu.cn.

$\dagger$ Supplemental material for this article may be found at http://jvi .asm.org/.

${ }^{\nabla}$ Published ahead of print on 18 June 2008.
}

sociated with a wide range of acute or chronic respiratory syndromes $(3,4,7-9,11,12,15,20,22,35,39,40,47)$. In the wake of the SARS outbreak, several novel HCoVs have been discovered, one of which is HCoV-HKU1 (9, 39). HCoVHKU1 has achieved global distribution since it was first identified in 2005: infections were first characterized in Hong Kong (26), followed by the identification of several strains of the virus in Korea (9), Europe (5, 17), Australia (31), and North America (14). In contrast to the lethal SARS-CoV, infection by HCoV-HKU1 usually leads to self-limiting syndromes affecting the lower respiratory tract. Nevertheless, the consequences could be more severe in patients with a compromised or immature immune system, such as asthma sufferers or newborn infants (24). Genome sequencing has confirmed that the $\mathrm{HCoV}-\mathrm{HKU} 1$ virus belongs to $\mathrm{CoV}$ group $2 \mathrm{~A}$ and shares high sequence homology with MHV and BCoV (39).

The functional components of the $\mathrm{CoV}$ replication machinery are released via posttranslational cleavage by two or three proteases. These proteases were first designated the papainlike protease (PLP) and 3C-like protease (3CL) for their respective sequence homology to the papain and rhinovirus $3 \mathrm{C}$ proteases. The $3 \mathrm{CL}$ protease also is commonly known as the main protease $\left(\mathrm{M}^{\mathrm{pro}}\right)$ because of the major role it plays in the proteolytic pathway, which makes it the most attractive pharmacological target for anti-CoV drug design. CoV $\mathrm{M}^{\text {pro }}$ s have been intensively studied, and crystal structures have been determined for the $\mathrm{M}^{\text {pro }} \mathrm{s}$ from the following CoVs: HCoV strain 229E (HCoV-229E) (2), porcine TGEV (1), avian IBV (41), and SARS-CoV (44). These structures are representative of group 1 (HCoV-229E and TGEV), group 2B (SARS-CoV), and group 3 (IBV) CoVs. However, no structure of the $\mathrm{M}^{\text {pro }}$ from a group 2A CoV (MHV, HCoV-HKU1, and HCoV$\mathrm{OC} 43$ ) has been determined to date. The absence of structural data presents a major obstacle for structure-aided drug optimization targeting group $2 \mathrm{~A} \mathrm{CoVs.}$ 
The $\mathrm{M}^{\mathrm{pro}} \mathrm{s}$ from different $\mathrm{CoV}$ groups are homologous in both sequence and main-chain architecture. They share a similar substrate binding sequence, with a requirement for glutamine at the $\mathrm{P} 1$ position and a strong preference for leucine/ methionine at P2. Based on this information, broad-spectrum lead compounds (43) with micromolar $K_{i}$ values have been designed that target $\mathrm{CoV} \mathrm{M}^{\text {pro }}$ s. However, structural data for the $\mathrm{M}^{\text {pro }}$ s from classical group 2A CoVs still are not available, posing a problem for further optimization.

Although $\mathrm{CoV} \mathrm{M}^{\text {pro }} \mathrm{s}$ exhibit absolute specificity for glutamine in the $\mathrm{P} 1$ position, recent research (38) has shown that the $\mathrm{M}^{\text {pro }}$ from $\mathrm{HCoV}-\mathrm{HKU} 1$ may possess an unusual substrate preference at $\mathrm{P} 1$ site quite different from that of other $\mathrm{CoV}$ $\mathrm{M}^{\text {pro }}$ s. Here, we report the structure of HCoV-HKU1 $\mathrm{M}^{\text {pro, }}$, which serves as a model for group $2 \mathrm{~A} \mathrm{CoVs}$ in complex with a synthetic peptidomimetic inhibitor, N3. The structure and subsequent enzyme activity assays help to resolve the issue of the relative conservation at the $\mathrm{P} 1$ position based on genome sequencing. Moreover, this complex structure provides further structural data for rational drug design against HCoVs.

\section{MATERIALS AND METHODS}

cDNA and plasmid. The cDNA encoding HCoV-HKU1 $\mathrm{M}^{\text {pro }}$ was kindly provided by K. Y. Yuen from the Department of Microbiology, Hong Kong University, Hong Kong Special Administrative Region, China. BamHI and XhoI restriction sites were attached to the $5^{\prime}$ and $3^{\prime}$ ends separately by PCR, and the PCR product first was inserted into the pMD-18T vector (Takara). The DNA of interest then was cleaved from the $\mathrm{T}$ vector and subcloned into a glutathione $S$-transferase-tagged expression vector, pGEX-4T-1. The validity of the whole procedure was confirmed by DNA sequencing.

Protein expression and purification. The plasmid was first transformed into the commercial Escherichia coli strain BL21(DE3) Rosetta (Invitrogen). After incubation at $37^{\circ} \mathrm{C}$ overnight on an $\mathrm{Amp}^{+}$algae Luria-Bertani (LB) plate, fresh transformants were inoculated into $5 \mathrm{ml} \mathrm{LB}$ medium in the presence of 100 $\mu \mathrm{g} / \mathrm{ml}$ ampicillin. After growth for $12 \mathrm{~h}$, the incubation system was scaled up to 1 liter LB medium with the same concentration of antibiotics in a 2-liter flask, and the solution was shaken vigorously at $37^{\circ} \mathrm{C}$ until the optical density at $600 \mathrm{~nm}$ reached 0.6. Cells were induced by $0.5 \mathrm{mM}$ isopropyl- $\beta$-D-thiogalactopyranoside (Sigma) at $16^{\circ} \mathrm{C}$ overnight.

Cell pellets were harvested by centrifugation, resuspended in $40 \mathrm{ml}$ phosphatebuffered saline buffer with $2 \mathrm{mM}$ dithiothreitol and $7 \mathrm{mM} \beta$-mercaptoethanol, and sonicated on ice for $25 \mathrm{~min}$. The supernatant was collected after the centrifugation of the sonicant at $15,000 \mathrm{rpm}$ for $40 \mathrm{~min}$.

Affinity purification was achieved by letting the supernatant flow through $2 \mathrm{ml}$ glutathione $S$-transferase affinity medium twice. On-column digestion lasted for $16 \mathrm{~h}$ at $4^{\circ} \mathrm{C}$ with thrombin (New England BioLabs), and the protein of interest was harvested and concentrated to $30 \mathrm{mg} / \mathrm{ml}$. The N3 inhibitor then was added to a final molar ratio of $1: 1$ and incubated at $4^{\circ} \mathrm{C}$ overnight. Finally, the $\mathrm{HCoV}$ HKU1 $\mathrm{M}^{\text {pro }}$-inhibitor complex was purified by gel filtration using a Superdex 200 (10/30) column (GE Healthcare). The protein concentration was adjusted to 20 $\mathrm{mg} / \mathrm{ml}$ for crystallization trials.

Crystallization and structure determination. Crystals of $\mathrm{HCoV}-\mathrm{HKU} 1 \mathrm{M}^{\text {pro }}$ were grown in $0.1 \mathrm{M}$ imidazole, $\mathrm{pH} 6.0$, and $0.6 \mathrm{M}$ sodium acetate by the hanging drop vapor diffusion method. Synchrotron X-ray diffraction data were collected on beamline BL-5A of the Photon Factory (Tsukuba, Japan) and processed to 2.5- $\AA$ resolution, using HKL2000 (29) for data indexing and scaling. Molecular replacement using the SARS $\mathrm{M}^{\text {pro }}$ structure (Protein Data Bank entry 2AMQ; $48 \%$ identity) as a template was performed with PHASER (32). The manual rebuilding of the structure was performed using Coot (13), and the structure was refined using REFMAC in the CCP4 suite (10). Final modification was carried out using CNS (6). The volume of the S1 cavity was calculated using VOIDOO (25).

Enzyme activity assays. Substrates and analogs were designed through three rounds of affinity optimization (42) by substrate mimicry and from a library of substrate analogs. The substrate and analogs were synthesized by Dawei Ma from the Institute of Organic Chemistry, Chinese Academy of Sciences, Shanghai, China.

The strategy employed for enzyme activity assays of HCoV-HKU1 $\mathrm{M}^{\text {pro }}$ has been described previously (43). Activity assays for HCoV-HKU1 M $\mathrm{M}^{\text {pro }}$ against the $\mathrm{CoV}$ consensus substrate and the HCoV-HKU1-specific substrate followed a similar protocol, which is described briefly below. The consensus substrate and HCoV-HKU1-specific substrates were fluorescent compounds with the sequences MCA-AVLQSGFR-Lys(Dnp)-Lys-NH $\mathrm{NH}_{2}$ and MCA-PRLHCTTNLys(Dnp)-Lys- $\mathrm{NH}_{2}$, respectively (greater than 95\% purity; GL Biotech Shanghai Ltd., Shanghai, China). A P1 single-mutant substrate also was synthesized with sequence MCA-AVLHSGFR-Lys(Dnp)-Lys- $\mathrm{NH}_{2}$.

The excitation and emission wavelengths of the fluorescent substrates were 320 and $405 \mathrm{~nm}$, respectively. A buffer consisting of $50 \mathrm{mM}$ Tris- $\mathrm{HCl}$ (pH 7.3) and $1 \mathrm{mM}$ EDTA was used for enzyme activity assays at $30^{\circ} \mathrm{C}$. The reaction was initiated by adding protease (final concentration, $2 \mu \mathrm{M}$ ) to a solution containing different final concentrations of the substrate $(3.2$ to $40 \mu \mathrm{M})$. Strict kinetic parameters for the inhibition assay were determined according to the previously reported protocol (43). All results from enzyme activity assays were calculated using data based on at least three independent parallel experiments.

Coordinate accession number. Coordinates and structure factors for the HKU1 $\mathrm{M}^{\text {pro }}$ in complex with inhibitor N3 have been deposited in the Protein Data Bank under entry ID 3D23.

\section{RESULTS}

Structural overview. Four protein molecules (denoted A, B, C, and D) occupy one asymmetric unit, with one N3 molecule per protomer. Two of the protomers form a typical homodimer, while the remaining two protomers dimerize with their adjacent symmetry-related counterparts (Fig. 1a). Each protomer exhibits a three-domain (I to III) architecture that is common to other CoV M ${ }^{\text {pro }}$ structures $(1,2,42,44)$ : domains I and II have chymotrypsin-like folds, and domain III displays a globular $\alpha$-helical cluster that is unique to $\mathrm{CoV} \mathrm{M}^{\mathrm{pro}}$. The catalytic site, including the Cys-His dyad, and the relatively shallow substrate binding pocket of $\mathrm{HCoV}-\mathrm{HKU} 1 \mathrm{M}^{\text {pro }}$ are located in the cleft between domains I and II. The substratebinding pocket features two deeply buried sites (P1 and P2) and several sites with different levels of solvent exposure (P3, P4, and P5) (Fig. 1b). X-ray data collection and refinement statistics are summarized in Table 1.

Michael acceptor and catalytic dyad. Clear and continuous electron density was observed between the reactive backbone carbon atom of the N3 substrate and the S $\gamma$ atom of Cys145 in the inhibitor-bound HCoV-HKU1 $\mathrm{M}^{\text {pro }}$ structure. We conclude that this reaction can be categorized as an electrophilic addition mediated by a Michael acceptor, obeying the $K_{i}-k_{3}$ kinetics, where $K_{i}$ is the dissociation constant and $k_{3}$ is the turnover number, according to the following scheme:

$$
E+S \stackrel{K_{m}}{\longleftrightarrow} E S \stackrel{k_{\text {cat }}}{\longrightarrow} \mathrm{E}-\mathrm{S}
$$

As the covalently bound inhibitor is a mimic of the real peptide substrate, it is possible to model the transition state by treating the enzyme-inhibitor complex structure as a snapshot of the catalytic dyad, and hence to predict parameters of the $K_{m}$ $k_{\text {cat }}$ kinetics, according to the following scheme:

$$
E+I \stackrel{K_{i}}{\longleftrightarrow} E I \stackrel{k_{3}}{\longrightarrow} E-I
$$

This catalytic dyad involves residues His41 and Cys145, and the intermediate state might be stabilized by the oxyanion hole (28) formed by the backbone amides of the oxyanion loop from Phe140 to Cys145 (Fig. 2a). The oxyanion hole is crucial to the 

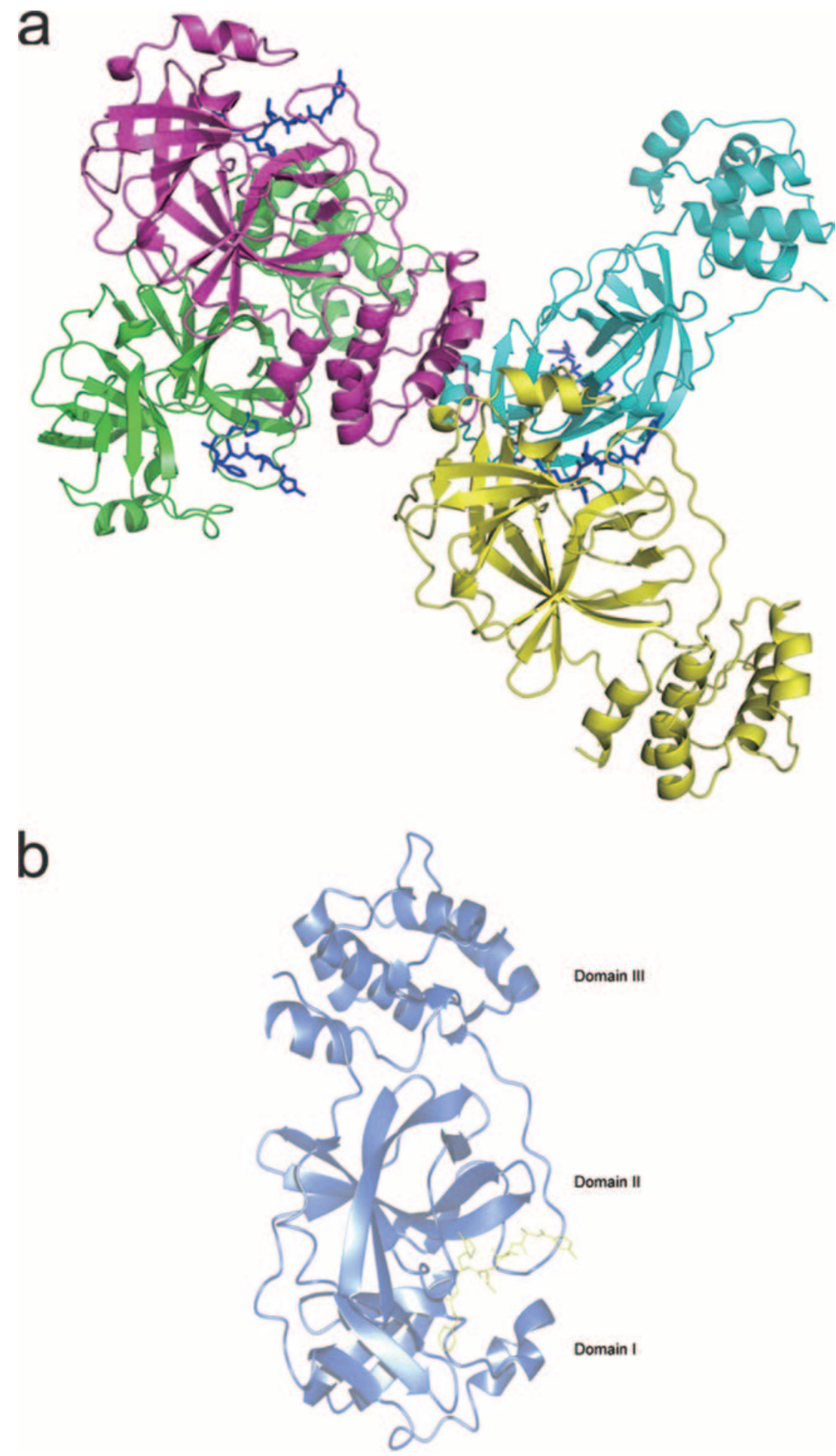

FIG. 1. (a) Structural overview of four protomers (A, green; B, cyan; C, magenta; and D, yellow) in one asymmetric unit, represented as cartoons. N3 inhibitors are shown as blue sticks. (b) Structural overview of the enzyme-inhibitor complex of one monomer unit. The main chain of the enzyme is represented as blue cartoons, and the synthetic inhibitor is shown as yellow sticks. The three domains are labeled.

stabilization of the intermediate state, so the formation of the oxyanion hole has a significant influence on $k_{\text {cat }}$. As discussed for the inhibitor design targeting human rhinovirus $3 \mathrm{C}$ proteases (28), the correct organization of this oxyanion loop also is essential to the $k_{3}$ step for mechanism-based suicide inhibitors.

Surrounding the S $\gamma$ atom of Cys145, we observe well-defined amides from the loop from residues 142 to 145 . Similarly to 
TABLE 1. X-ray data-processing and refinement statistics

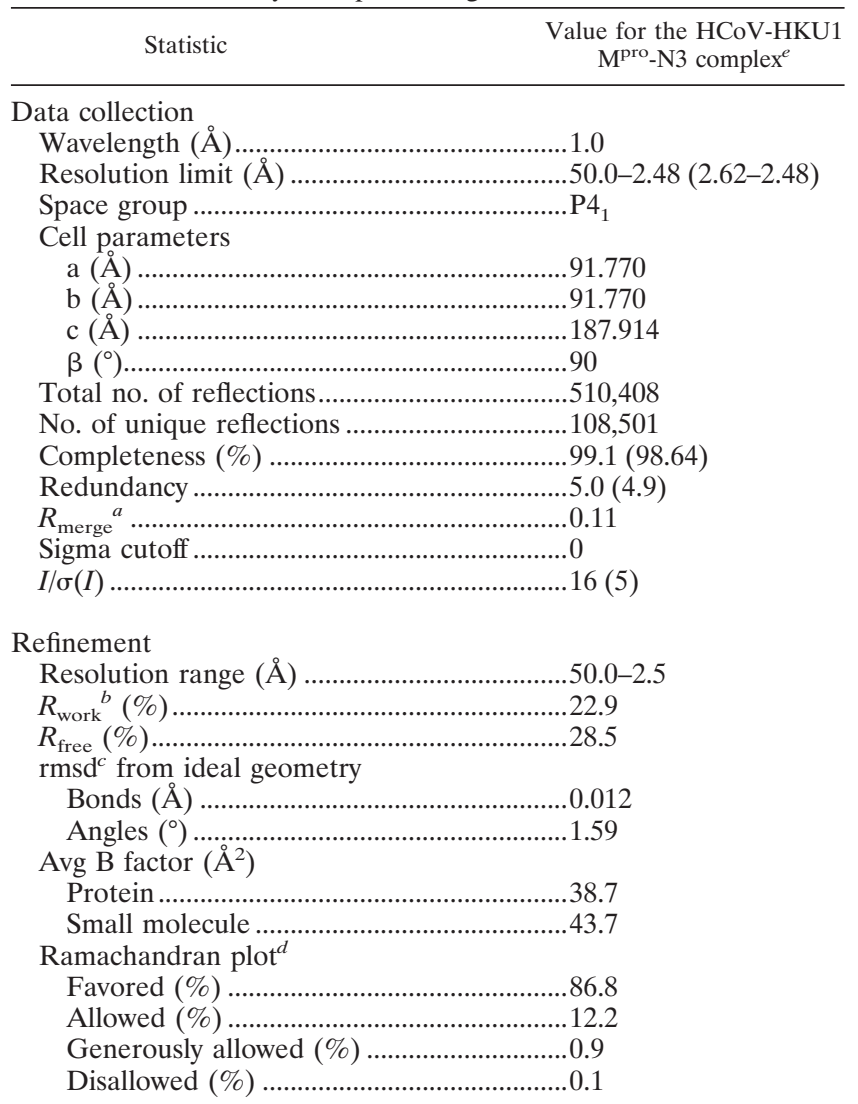

${ }^{a} R_{\text {merge }}=\Sigma I_{i}-<I>/ \Sigma I$, where $I_{i}$ is the intensity of an individual reflection and $\langle I\rangle$ is the average intensity of that reflection.

${ }^{b} R_{\text {work }}=\Sigma F_{p}-F_{c} / \Sigma F_{p}$, where $F_{c}$ is the calculated and $F_{p}$ is the observed structure factor amplitude.

${ }^{c} \mathrm{rmsd}$, root mean squares deviation.

${ }^{d}$ Ramachandran plots were generated by using the program PROCHECK.

${ }^{e}$ Numbers in parentheses correspond to the highest-resolution shell.

human rhinovirus $3 \mathrm{C}$ proteases (28), these amide dipoles construct a tetrahedral oxyanion hole. From native and complex structures of SARS-CoV M $\mathrm{M}^{\text {pro }}$, the correct orientation of these backbone amides is triggered and maintained by substrate binding, in particular by the binding of the $\mathrm{P} 1$ residue and interaction between the $\mathrm{N}$ finger and the substrate $(2,44)$, in which the backbone carbonyl of Leu141 is hydrogen bonded to the side chain oxygen of Ser144. The correct position of Leu141 is maintained by a hydrogen bond between the carbonyl group of Phe140 and the amide group of the P1 side chain and by hydrophobic stacking between His163 and Phe140. Although the analysis of the HCoV-HKU1 M ${ }^{\text {pro }}$ structure in complex with $\mathrm{N} 3$ (Fig. 2a) shows that the P1 side chain exerts no direct influence on the residues forming the oxyanion hole, its side chain oxygen atom forms a strong hydrogen bond (2.6 $\AA$ ) with His163 and helps to strengthen the stacking interaction with Phe140. Furthermore, the nitrogen atom of the P1 side chain also forms a hydrogen bond (3.1 $\AA$ ) with the backbone of Phe140, thus helping to maintain the oxyanion loop (Phe140-Cys145) in its proper conformation. For the above reasons, we conclude that the $\mathrm{P} 1$ side chain is important for the network of interactions stabilizing the oxyanion hole.
The $\mathrm{S} 1$ pocket has a smaller size to accommodate $\mathrm{P} 1$ histidine. Given its crucial role in the catalytic process, glutamine outperforms other residues as the signature of the $\mathrm{M}^{\text {pro }}$ substrate at the $\mathrm{P} 1$ position. In addition to this advantage, the side chain of glutamine in the P1 position suitably fits with residues forming the S1 subsite via Van der Waals interactions (Fig. 2b). From the HCoV-HKU1 genome sequence, 11 out of 12 $\mathrm{M}^{\text {pro }}$ recognition sites have Gln at the $\mathrm{P} 1$ position. In our structure, the N3 molecule has a lactam ring as an analog to the glutamine residue (the cross-linking between the $\mathrm{C} \gamma$ and $\mathrm{N}$ atoms helps to select the stretching conformation from the ensemble of rotamers and better occupy the binding cleft). In $\mathrm{HCoV}-\mathrm{HKU} 1 \mathrm{M}^{\text {pro }}$ structures, the lactam ring protrudes into the $\mathrm{S} 1$ pocket via a hydrogen bond to the imidazole ring $\mathrm{NH}$ of His162 at a distance of $2.6 \AA$. However, unlike the SARS-CoV $M^{\text {pro }}$ structure in complex with N3, the NH of the HKU1-N3 lactam ring fails to recruit a water molecule to satisfy a second $\mathrm{S} 1$ hydrogen bond. Instead, the $\mathrm{N}$-terminal $\mathrm{O} \gamma$ atom might

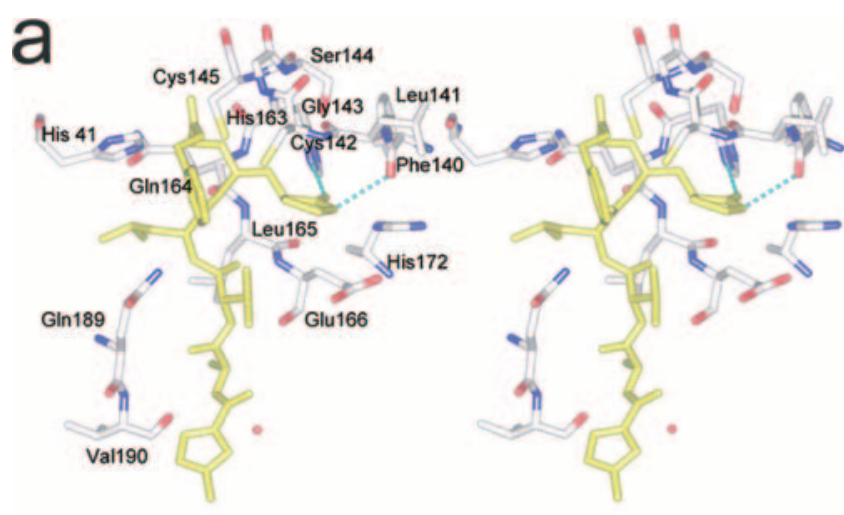

b

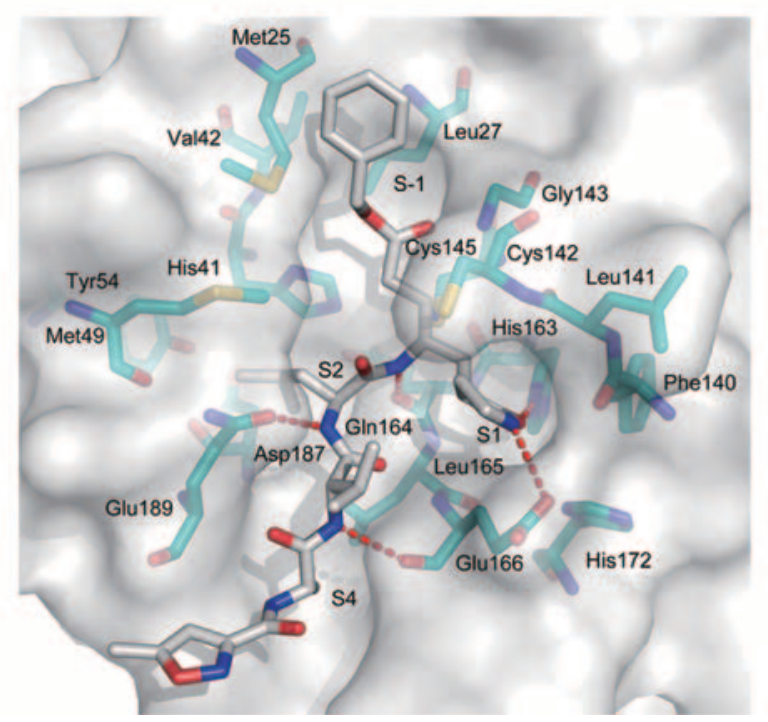

FIG. 2. (a) Details of the interaction between the P1 side chain and the defined oxyanion loop, shown in stereo representation. Side chains are shown as sticks, and the crucial hydrogen bond between His163 and the substrate side chain is shown by a cyan dashed line. (b) Details of the substrate-binding pocket. The inhibitor is shown in the following color scheme: $\mathrm{C}$, white; $\mathrm{O}$, red; and $\mathrm{N}$, blue. The crucial residues of the enzyme are shown in the following color scheme: C, cyan; O, red; N, blue; and S, yellow. Hydrogen bonds are shown as red dashed lines. 
TABLE 2. Activity assay of HCoV-HKU1

\begin{tabular}{lcc}
\hline Substrate $^{a}$ & $K_{m}(\mu \mathrm{M})$ & $k_{\text {cat }}$ \\
\hline C & $83.2 \pm 13.3$ & $1.1 \pm 0.12$ \\
H & $22.3 \pm 5.2$ & $0.09 \pm 0.013$ \\
SMC & $265.3 \pm 21.5$ & $0.03 \pm 0.001$ \\
\hline
\end{tabular}

${ }^{a}$ Substrate $\mathrm{C}$ refers to the consensus substrate of $\mathrm{CoV} \mathrm{M}^{\text {pro }}$ s with the sequence MCA-AVLQSGFR-Lys(Dnp)-Lys- $\mathrm{NH}_{2}$. Substrate $\mathrm{H}$ refers to the specific substrate of HCoV-HKU1, including a mutation at the P1 site from glutamine to histidine, with the sequence MCA-PRLHCTTN-Lys(Dnp)-Lys-NH Substrate SMC refers to the consensus substrate, including a mutation at the P1 site from glutamine to histidine, with the sequence MCA-AVLHSGFRLys(Dnp)-Lys- $\mathrm{NH}_{2}$.

provide a weak electronegative interaction to stabilize the $\mathrm{NH}$ atom; the interaction likely is stronger due to the presence of redundant residues as a cloning artifact, hindering the $\mathrm{N}$-terminal Ser from coming any closer to the $\mathrm{NH}$ of $\mathrm{P} 1$ side chain.

Nevertheless, compared with the $\mathrm{M}^{\mathrm{pro}} \mathrm{s}$ from other $\mathrm{CoV}$ groups, the structure of the HCoV-HKU1 $\mathrm{M}^{\text {pro }}$ has an S1 pocket with a relatively smaller volume of $\sim 18.1 \AA^{3}$. In contrast, the volume of the S1 pocket of TGEV M $\mathrm{M}^{\text {pro }}$ is $\sim 19.1 \AA^{3}$, that of of IBV M $\mathrm{M}^{\text {pro }}$ is $\sim 21.7 \AA^{3}$, and that of SARS-CoV $\mathrm{M}^{\text {pro }}$ is $\sim 19.5 \AA^{3}$. The reduced size of the $\mathrm{S} 1$ pocket might be caused by the position of the loop Leu167-Cys171, which is bent upward by about $90^{\circ}$. As a result, the smaller S1 pocket might tolerate mutation to short-chain residues at the $\mathrm{P} 1$ position, in which case a weakened oxyanion hole is to be expected. Novel substrate specificity already has been found in the HCoVHKU1 genome, in which the $\mathrm{M}^{\text {pro }}$ recognition site between the helicase and exonuclease utilizes histidine instead of glutamine at the P1 position. Mimicking proteolysis in the cell, enzyme activity assays using a synthetic fluorogenic substrate confirm the existence of such a cleavage event in vitro and exhibit novel enzymatic properties not seen with the consensus substrate (Table 2).

Enzyme activity assays indicate that the affinity for a substrate containing a single mutation at the $\mathrm{P} 1$ position decreases to $30 \%$ of the affinity for the native consensus substrate, which can be attributed to the loss of a hydrogen bond resulting from the mutation of glutamine to histidine. The scissile velocity decreases to $3 \%$, which is to be expected, since the histidine residue lacks an oxygen atom that is required to form a strong hydrogen bond and support the intermediate oxyanion hole. However, when determining the influence of P2-P5 variance in the HCoV-HKU1-specific substrate, we observed an unusual fourfold elevation in the $K_{m}$ compared to that of the consensus substrate and a minor rescue of the scissile velocity (a threefold elevation of the single-mutant substrate). This could be explained by the contribution of the non-P1 residues in the HCoV-HKU1-specific substrate, since activity assays for the single-mutant substrate imply that mutation at the P1 position has a detrimental effect not only on the substrate binding affinity but also on the substrate scissile velocity.

The S2 pocket presents group-specific features but no group-specific substrate preferences. The $\mathrm{P} 2$ side chain of the ligand protrudes into the S2 pocket via interactions with the hydrophobic side chains of Met25, Pro52, and Tyr54 (Fig. 2B). The lid of the pocket is covered by a short $3_{10}$ helical region from Ser45-Asn51. To compare the diversity of the $\mathrm{S} 2$ pockets of all three $\mathrm{CoV}$ groups, the backbones of $\mathrm{M}^{\text {pro }}$ complex struc-

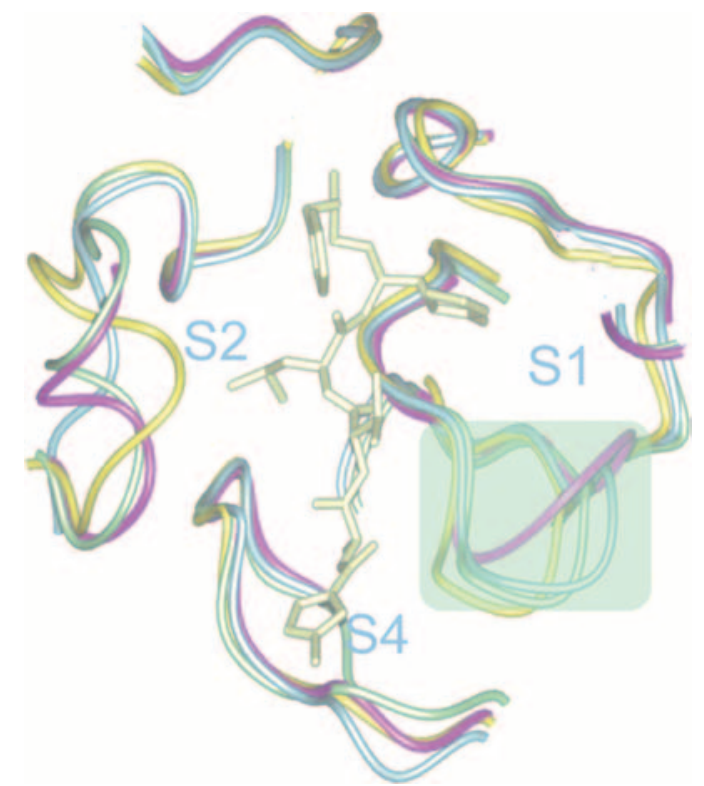

FIG. 3. S1 and S2 binding sites of HCoV-HKU1 $\mathrm{M}^{\text {pro }}$ main chains of four $\mathrm{M}^{\text {pro }}$ structures are superimposed and displayed in the neighborhood of the substrate-binding site. The $\mathrm{S} 1$ and $\mathrm{S} 2$ binding sites are highlighted by light green shadows. The main chains are represented in worm forms. Different colors are used to represent the strain of CoV. Lemon, synthetic compound; magenta, HCoV-HKU1; light green, SARS-CoV; light blue, TGEV; and yellow, avian IBV.

tures from all groups were superimposed (Fig. 3): for group 1, TGEV $M^{\text {pro }}$ in complex with the inhibitor N1, an ancestor of $\mathrm{N} 3$; for group 3, IBV $\mathrm{M}^{\text {pro }}$ in complex with N3; and for group 2B, SARS-CoV in complex with inhibitor N3. We observed three modes of secondary structure: the $3_{10}$ helix (HCoVHKU1 and SARS-CoV), a loose loop (IBV), and a tight loop (TGEV). Interestingly, the clustering of the secondary structure correlates with the temporary classification of CoVs. We then explored the natural recognition sequences to examine whether the group-specific features could result in different substrate specificities at the P2 site (Table 3). After summarizing the $\mathrm{P} 2$ residue type in the protease recognition sites of the HKU1 PP1ab genome, we observe that $\mathrm{M}^{\text {pro }}$ s prefer a hydrophobic residue at this position, which is also the case for SARS-CoV, IBV, and TGEV. Although there are a few ex-

TABLE 3. P2 residues in different $\mathrm{CoV}$ genomes

\begin{tabular}{ccccc}
\hline \begin{tabular}{c} 
M $\begin{array}{c}\text { pro cleavage } \\
\text { site no. }\end{array}$ \\
\cline { 2 - 5 }
\end{tabular} & SARS-CoV & HCoV-HKU1 & IBV & TGEV \\
\hline 1 & $\mathrm{~L}$ & $\mathrm{~L}$ & $\mathrm{~L}$ & $\mathrm{~L}$ \\
2 & $\mathrm{~F}$ & $\mathrm{~L}$ & $\mathrm{~L}$ & $\mathrm{~N}$ \\
3 & $\mathrm{~V}$ & $\mathrm{I}$ & $\mathrm{M}$ & $\mathrm{V}$ \\
4 & $\mathrm{~L}$ & $\mathrm{~L}$ & $\mathrm{~L}$ & $\mathrm{~L}$ \\
5 & $\mathrm{~L}$ & $\mathrm{M}$ & $\mathrm{L}$ & $\mathrm{L}$ \\
6 & $\mathrm{~L}$ & $\mathrm{~L}$ & $\mathrm{~L}$ & $\mathrm{~L}$ \\
7 & $\mathrm{M}$ & $\mathrm{V}$ & $\mathrm{V}$ & $\mathrm{M}$ \\
8 & $\mathrm{~L}$ & $\mathrm{M}$ & $\mathrm{L}$ & $\mathrm{L}$ \\
9 & $\mathrm{~L}$ & $\mathrm{~L}$ & $\mathrm{~L}$ & $\mathrm{~L}$ \\
10 & $\mathrm{~L}$ & $\mathrm{~L}$ & $\mathrm{~L}$ & $\mathrm{~L}$ \\
11 & $\mathrm{~L}$ & $\mathrm{M}$ & $\mathrm{L}$ & $\mathrm{L}$ \\
\hline
\end{tabular}



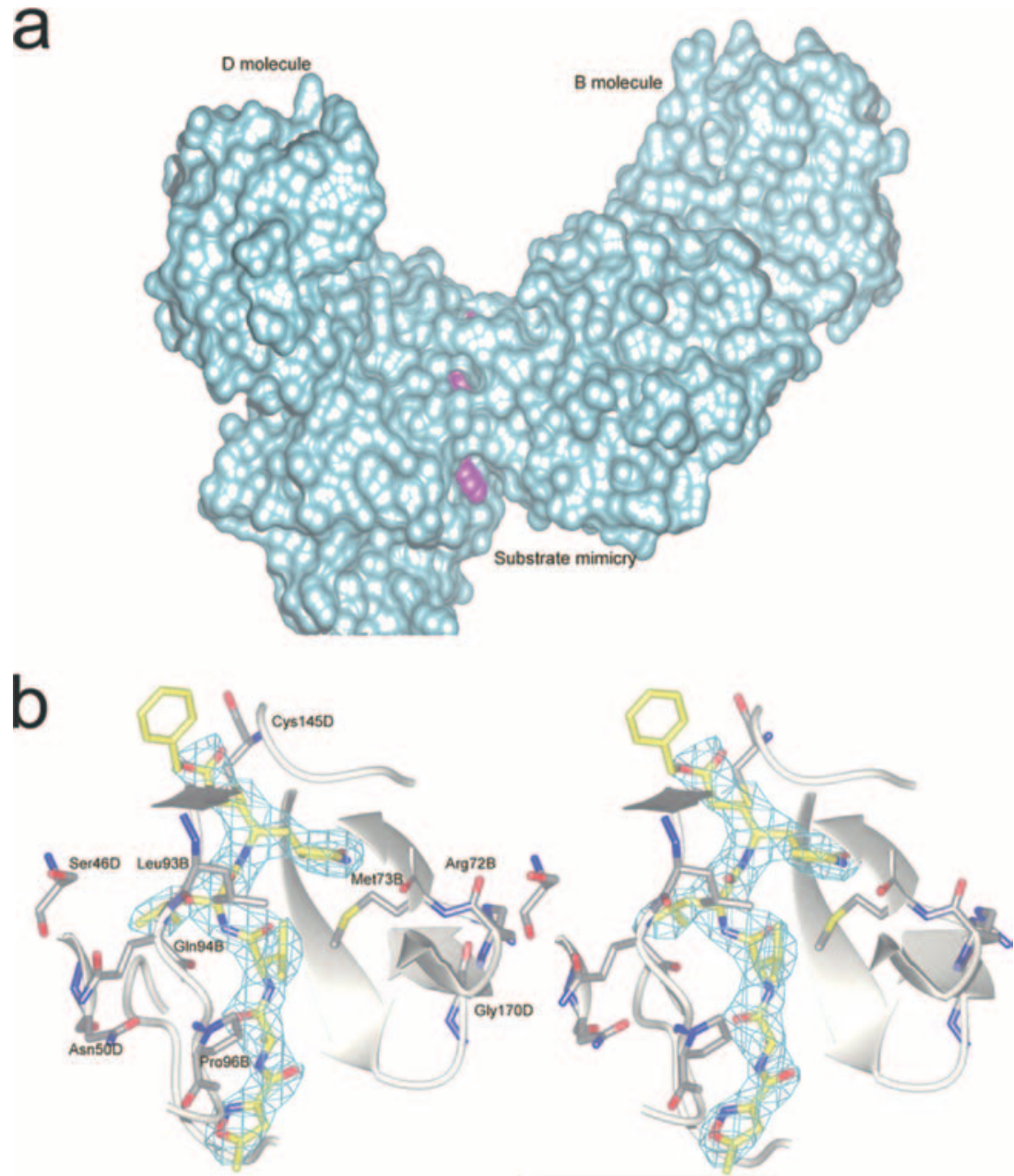

FIG. 4. (a) Overview of the P3 pocket. The inhibitor resides between the interface of molecules B and D. A cyan surface model is shown covering protomers B and D. The inhibitor is shown in magenta. (b) P3 interaction site of substrate in detail. Neighboring residues within 4 A of the S3 site are colored green. An Fo-Fc map contoured at $1.5 \sigma$ around the inhibitor is displayed in cyan. For the inhibitor, C atoms are colored yellow, $\mathrm{N}$ atoms are colored blue, and $\mathrm{O}$ atoms are colored red. The protein carbon atoms are colored gray. Neighboring main chains are displayed as white ribbons.

ceptions, such as asparagine or valine residues, leucine/methionine are the most abundant. This is consistent with the observation from our structure that the hydrophobic P2 side chain extends into the deep S2 site without clashing with the Van der Waals surface of the pocket (Fig. 2b). Therefore, on the one hand, considering the flexibility of S2 pocket as well as the residual space after occupation by the $\mathrm{P} 2$ residue, the optimal choice for leucine or methionine might be related to the size of the S2 pocket. On the other hand, the similar preferences on $\mathrm{S} 2$ sites among group $1,2 \mathrm{a}, 2 \mathrm{~b}$, and $3 \mathrm{CoV}$ $\mathrm{M}^{\text {pro }}$ s does challenge the efficacy of designing group-specific inhibitors by altering only $\mathrm{P} 2$ moieties.

The $\mathrm{P} 3$ position. Two out of four protomers in one asymmetric unit exhibit a solvent-exposed P3 side chain, which may interact weakly with the edge of the substrate-binding cleft via Van der Waals forces (Fig. 2b). To assess whether P3 side chain variation can influence the potency of inhibition, we synthesized a small library of six inhibitors (see Table S1 in the supplemental material) to assay their affinity by the second-order reaction coefficients. The results are summarized in Fig. S1 in the supplemental material. It appears unlikely that the preference can be attributed exclusively to weak interactions. When investigating other possible P3-related interactions found in our HCoV-HKU1 $\mathrm{M}^{\text {pro }}$ structure, we scrutinized and evaluated the mainly hydrophobic structural interaction (Fig. 4a) between molecules B and D (Fig. $4 \mathrm{~b})$. From the crystal packing, we observed a crystallographic contact close to the P3 position (see Fig. S3 in the supplemental material) that we suspect might affect this property of the P3 residue. To examine the physiological relevance, we conducted dynamic light-scattering experiments to check for higher-molecular-weight states that should be expected in solution if this interaction is related to one of the stable physiological states. However, dynamic light-scattering experiments did not identify a tetramer or higher-molecular-weight complex in aqueous solution (see Fig. S2 in the supplemental material). Thus, the substrate selectivity at the P3 position may be attributed to other weak factors, such as solvent-side chain interactions and Van der Waals interactions with the substrate binding cleft, rather than to a strong and direct interaction, which is more likely to be influenced by crystal contacts. 
a
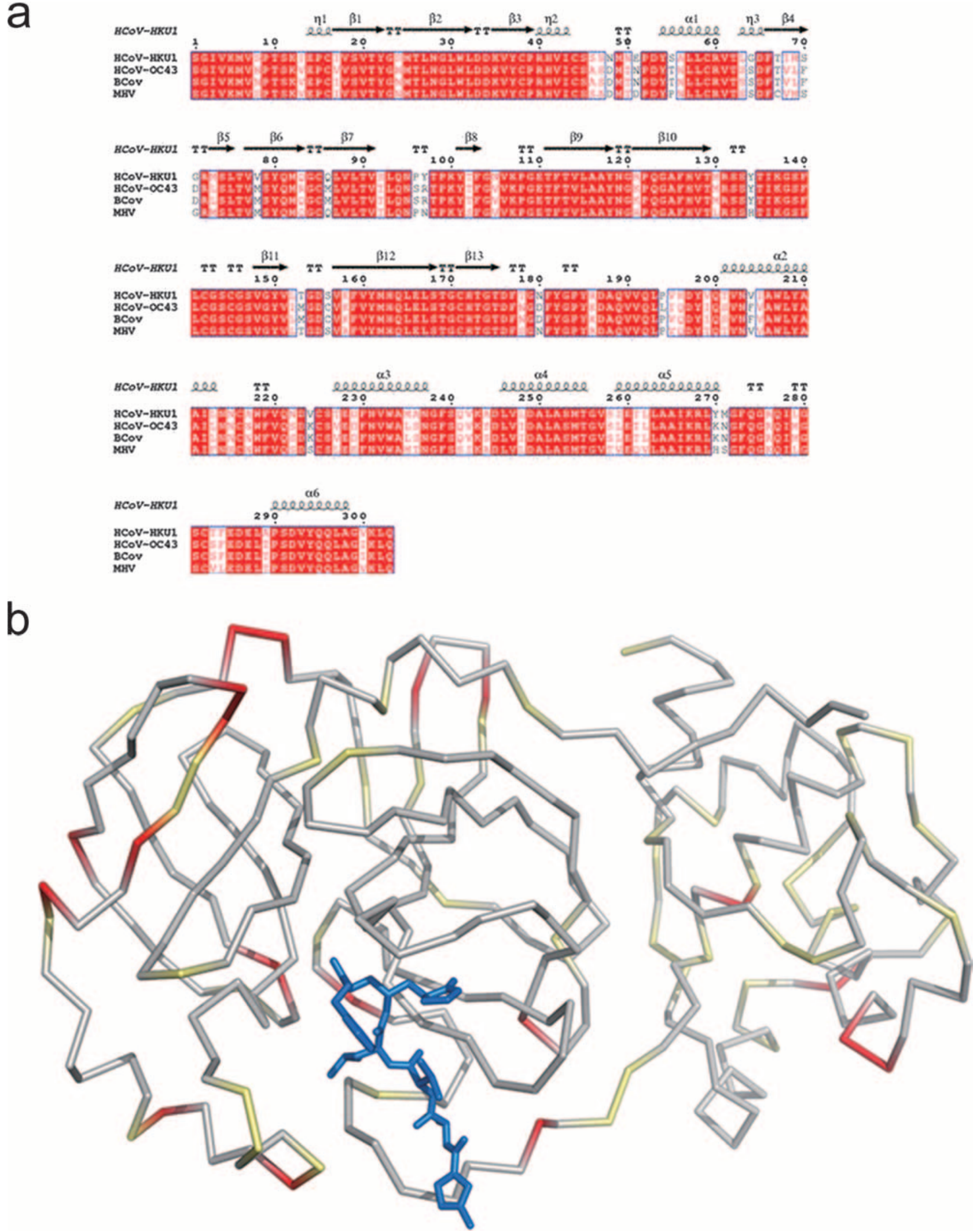

FIG. 5. (a) Sequence alignment of a typical $\mathrm{M}^{\text {pro }}$ from $\mathrm{CoV}$ group $2 \mathrm{~A}$ exhibits high homology. The alignment was performed with ClustalW (33), and the final figure was generated with ESPript1.0 (18). White letters with red backgrounds refer to identical residues, red letters with white backgrounds refer to conservative variation, and black letters with white backgrounds refer to nonconservative mutations. (b) Three-dimensional representation of nonconserved mutations in group $2 \mathrm{~A} \mathrm{CoV} \mathrm{M}^{\text {pro }}$ s mapped onto the HCoV-HKU1 $\mathrm{M}^{\text {pro }}$ structure. Identical residues are colored white, conserved mutations are colored yellow, and nonconserved mutations are colored red. The inhibitor is colored blue. 


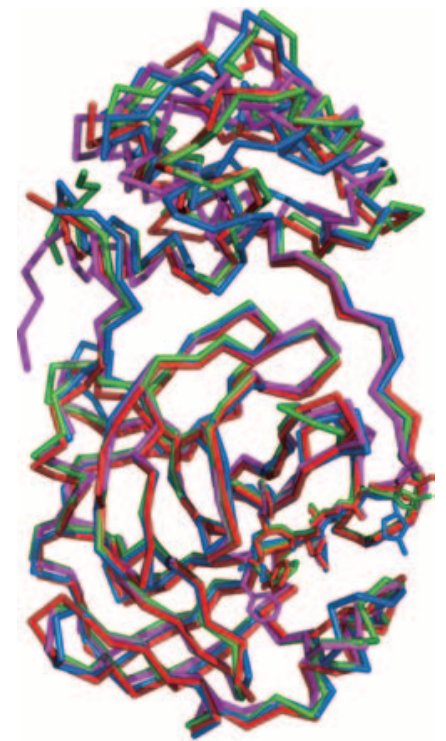

FIG. 6. Superposition of representative $\mathrm{CoV} \mathrm{M}^{\text {pro }}$ in complex with Michael acceptor-based inhibitors of group 1 (TGEV; blue), group 2A (HCoV-HKU1; green), group 2B (SARS-CoV; magenta), and group 3 (IBV; red). The color of each inhibitor is consistent with that of its host.

\section{DISCUSSION}

The HKU1 structure is a suitable model for group $2 \mathrm{~A} \mathrm{CoV}$ $\mathbf{M}^{\text {pro }} \mathbf{s}$. The crystal structure of $\mathrm{M}^{\text {pro }}$ from HCoV-HKU1 is the first to be determined from a group $2 \mathrm{~A} \mathrm{M}^{\text {pro }}$. Since members of the group 2A CoVs share particularly high sequence identity (4) (Fig. 5a), nonconservative changes occur mainly in flexible regions of the $\mathrm{HCoV}-\mathrm{HKU} 1 \mathrm{M}^{\text {pro }}$ structure, including domain linkages and the molecular surface. Notable variations from residues 46 to 71 in group $2 \mathrm{~A}$ sequences are located in or nearby the $\mathrm{S} 2$ pocket, which might infer properties relating to enzyme activity. However, since even greater differences between the different groups of CoVs exhibit no particular enzyme-specific preferences in the $\mathrm{S} 2$ pocket, the relatively small variations here may be unlikely to challenge the consensus substrate preference among CoVs at the P2 position. The residue ranges 20 to 40,140 to 160 , and 187 to 189 , as well as residue 166 , are highly conserved and are involved in the formation of the S-1 and S1 pockets, together with the walls of the binding pocket of the $\mathrm{P} 3$ side chain. Thus, it is reasonable to conclude that the HCoV-HKU1 $\mathrm{M}^{\text {pro }}$ structure is a suitable model for the study of group 2A CoVs, both in terms of enzyme activity and inhibitor design.

Michael acceptor inhibitors interact with $\mathrm{CoV} \mathrm{M}^{\text {pro }}$ in a similar manner. As Michael acceptor suicide inhibitors, N3 and its derivatives cocrystallize with the $\mathrm{CoV} \mathrm{M}^{\text {pro }}$ in a similar manner (Fig. 6). The backbones of the peptidomimetic compounds align antiparallel to the $\beta$-strands, constituting the binding cleft. The $\mathrm{P} 1$ and $\mathrm{P} 2$ residues fit into the $\mathrm{S} 1$ and $\mathrm{S} 2$ pockets, respectively, and have a major contribution to substrate preference: glutamine at P1 and leucine/methionine at $\mathrm{P} 2$. In our future optimization of $\mathrm{M}^{\text {pro }}$ inhibitors, we think that the glutamine (or its analog) might be worth keeping in the P1 position, while it would be reasonable to conduct a comparison of leucine to methionine for the evaluation of the $\mathrm{P} 2$ residue. Aside from these deeply buried side chains, the solvent-exposed P3 provides no straightforward information for the substrate-enzyme interaction, though the variation at this position shows an obvious impact on inhibition. Alternatively, we might employ random screening for further optimization at the P3 position.

Conclusions. Structural data now are available for $\mathrm{CoV}$

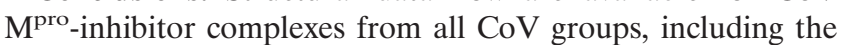
two subgroups of the group $2 \mathrm{CoVs}$. Moreover, these structures provide further confirmation for the efficacy of widespectrum inhibitors at atomic resolution. From enzyme activity assays, we succeeded in identifying the atypical substrate specificity of HCoV-HKU1 $\mathrm{M}^{\text {pro }}$ with higher affinity $\left(K_{m}\right)$ and lower reactivity $\left(k_{\text {cat }}\right)$ than those of the consensus $\mathrm{CoV} \mathrm{M}^{\text {pro }}$ substrate. We attributed these properties to the contribution of non-P1 residues and the distortion of the oxyanion hole. Although the S2 pockets from different groups share groupspecific features, an investigation of the natural recognition sequences does not find different residue-type specificity at the P2 site.

Considering the high identity shared by group $2 \mathrm{~A} \mathrm{CoVs}$, these structural features of HCoV-HKU1 $\mathrm{M}^{\text {pro }}$, together with corresponding enzyme activity assays, will help to profile HCoV-HKU1 and other newly emerging etiologic agents from this group of CoVs.

\section{ACKNOWLEDGMENTS}

We thank K. Y. Yuen for providing the cDNA of HCoV-HKU1 $\mathrm{M}^{\mathrm{pro}}$, as well as Zhiyong Lou and Xiaohang Tong for technical assistance and data collection for HCoV-HKU1 $\mathrm{M}^{\text {pro }}$ in complex with $\mathrm{N} 3$.

This work was supported by Project 973 of the Ministry of Science and Technology of China (grant numbers 2006CB806503 and 2007CB914301), the National Natural Science Foundation of China (grant numbers 30221003 and 30730022), the Sino-German Center [grant number GZ236(202/9)], the Sino-European Project on SARS Diagnostics and Antivirals (SEPSDA) of the European Commission (grant number 003831), and the Tsinghua University Ph.D. student innovation fund.

\section{REFERENCES}

1. Anand, K., G. J. Palm, J. R. Mesters, S. G. Siddell, J. Ziebuhr, and R. Hilgenfeld. 2002. Structure of $\mathrm{CoV}$ main proteinase reveals combination of a chymotrypsin fold with an extra alpha-helical domain. EMBO J. 21:32133224.

2. Anand, K., J. Ziebuhr, P. Wadhwani, J. R. Mesters, and R. Hilgenfeld. 2003. Coronavirus main proteinase (3CLpro) structure: basis for design of antiSARS drugs. Science 300:1763-1767.

3. Arden, K. E., M. D. Nissen, T. P. Sloots, and I. M. Mackay. 2005. New human coronavirus, HCoV-NL63, associated with severe lower respiratory tract disease in Australia. J. Med. Virol. 75:455-462.

4. Bastien, N., K. Anderson, L. Hart, P. Van Caeseele, K. Brandt, D. Milley, T. Hatchette, E. C. Weiss, and Y. Li. 2005. Human coronavirus NL63 infection in Canada. J. Infect. Dis. 191:503-506.

5. Bosis, S., S. Esposito, H. G. Niesters, E. Tremolati, S. Pas, N. Principi, and A. D. Osterhaus. 2007. Coronavirus HKU1 in an Italian pre-term infant with bronchiolitis. J. Clin. Virol. 38:251-253.

6. Brünger, A. T., P. D. Adams, G. M. Clore, W. L. DeLano, P. Gros, R. W. Grosse-Kunstleve, J. S. Jiang, J. Kuszewski, M. Nilges, N. S. Pannu, R. J. Read, L. M. Rice, T. Simonson, and G. L. Warren. 1998. Crystallography \& NMR system: a new software suite for macromolecular structure determination. Acta Crystallogr. D Biol. Crystallogr. 54:905-921.

7. Chang, L. Y., B. L. Chiang, C. L. Kao, M. H. Wu, P. J. Chen, B. Berkhout, H. C. Yang, and L. M. Huang. 2006. Lack of association between infection with a novel human coronavirus ( $\mathrm{HCoV}), \mathrm{HCoV}-\mathrm{NH}$, and Kawasaki disease in Taiwan. J. Infect. Dis. 193:283-286.

8. Chiu, S. S., K. H. Chan, K. W. Chu, S. W. Kwan, Y. Guan, L. L. Poon, and J. S. Peiris. 2005. Human coronavirus NL63 infection and other coronavirus infections in children hospitalized with acute respiratory disease in Hong Kong, China. Clin. Infect. Dis. 40:1721-1729. 
9. Choi, E. H., H. J. Lee, S. J. Kim, B. W. Eun, N. H. Kim, J. A. Lee, J. H. Lee, E. K. Song, S. H. Kim, J. Y. Park, and J. Y. Sung. 2006. The association of newly identified respiratory viruses with lower respiratory tract infections in Korean children, 2000-2005. Clin. Infect. Dis. 43:585-592.

10. Collaborative Computational Project, Number 4. 1994. The CCP4 suite: programs for protein crystallography. Acta Crystallogr. D Biol. Crystallogr. 50:760-763.

11. Dominguez, S. R., M. S. Anderson, M. P. Glode, C. C. Robinson, and K. V. Holmes. 2006. Blinded case-control study of the relationship between human coronavirus NL63 and Kawasaki syndrome. J. Infect. Dis. 194:1697-1701.

12. Ebihara, T., R. Endo, X. Ma, N. Ishiguro, and H. Kikuta. 2005. Detection of human coronavirus NL63 in young children with bronchiolitis. J. Med. Virol. 75:463-465.

13. Emsley, P., and K. Cowtan. 2004. Coot: model-building tools for molecular graphics. Acta Crystallogr. D Biol. Crystallogr. 60:2126-2132.

14. Esper, F., C. Weibel, D. Ferguson, M. L. Landry, and J. S. Kahn. 2006 Coronavirus HKU1 infection in the United States. Emerg. Infect. Dis. 12: 775-779.

15. Esposito, S., S. Bosis, H. G. Niesters, E. Tremolati, E. Begliatti, A. Rognoni, C. Tagliabue, N. Principi, and A. D. Osterhaus. 2006. Impact of human coronavirus infections in otherwise healthy children who attended an emergency department. J. Med. Virol. 78:1609-1615.

16. Garbino, J., S. Crespo, J. D. Aubert, T. Rochat, B. Ninet, C. Deffernez, W. Wunderli, J. C. Pache, P. M. Soccal, and L. Kaiser. 2006. A prospective hospital-based study of the clinical impact of non-severe acute respiratory syndrome (non-SARS)-related human coronavirus infection. Clin. Infect. Dis. 43:1009-1015.

17. Gerna, G., E. Percivalle, A. Sarasini, G. Campanini, A. Piralla, F. Rovida, E. Genini, A. Marchi, and F. Baldanti. 2007. Human respiratory coronavirus HKU1 versus other coronavirus infections in Italian hospitalised patients. J. Clin. Virol. 38:244-250.

18. Gouet, P., E. Courcelle, D. I. Stuart, and F. Metoz. 1999. ESPript: analysis of multiple sequence alignments in PostScript. Bioinformatics 15:305-308.

19. Granzow, H., U. Meyer, P. Solisch, E. Lange, and D. Fichtner. 1981. Morphology of coronaviruses-electron microscopic demonstration by negative contrast technic of the transmissible gastroenteritis virus of swine. Arch. Exp. Veterinarmed. 35:177-186.

20. Han, T. H., J. Y. Chung, S. W. Kim, and E. S. Hwang. 2007. Human coronavirus-NL63 infections in Korean children, 2004-2006. J. Clin. Virol. 38:27-31.

21. Hök, K. 1990. Demonstration of feline corona virus (FCV) antigen in organs of cats suspected of feline infectious peritonitis (FIP) disease. APMIS 98: 659-664.

22. Huang, I. C., B. J. Bosch, W. Li, M. Farzan, P. M. Rottier, and H. Choe. 2006. SARS-CoV, but not HCoV-NL63, utilizes cathepsins to infect cells: viral entry. Adv. Exp. Med. Biol. 581:335-338.

23. Ignatov, G., L. Vasileva, and K. Kharalambiev. 1979. Purification of coronaviruses from contaminated cattle feces. Vet. Med. Nauki 16:23-27.

24. Kistler, A., P. C. Avila, S. Rouskin, D. Wang, T. Ward, S. Yagi, D. Schnurr, D. Ganem, J. L. DeRisi, and H. A. Boushey. 2007. Pan-viral screening of respiratory tract infections in adults with and without asthma reveals unexpected human coronavirus and human rhinovirus diversity. J. Infect. Dis. 196: $817-825$

25. Kleywegt, G. J., and T. A. Jones. 1994. Detection, delineation, measurement and display of cavities in macromolecular structures. Acta Crystallogr. D Biol. Crystallogr. 50:178-185.

26. Lau, S. K., P. C. Woo, C. C. Yip, H. Tse, H. W. Tsoi, V. C. Cheng, P. Lee, B. S Tang, C. H. Cheung, R. A. Lee, L. Y. So, Y. L. Lau, K. H. Chan, and K. Y Yuen. 2006. Coronavirus HKU1 and other coronavirus infections in Hong Kong. J. Clin. Microbiol. 44:2063-2071.

27. Li, W., Z. Shi, M. Yu, W. Ren, C. Smith, J. H. Epstein, H. Wang, G. Crameri, Z. Hu, H. Zhang, J. Zhang, J. McEachern, H. Field, P. Daszak, B. T. Eaton, S. Zhang, and L. F. Wang. 2005. Bats are natural reservoirs of SARS-like coronaviruses. Science 310:676-679.

28. Matthews, D. A., P. S. Dragovich, S. E. Webber, S. A. Fuhrman, A. K. Patick L. S. Zalman, T. F. Hendrickson, R. A. Love, T. J. Prins, J. T. Marakovits, R. Zhou, J. Tikhe, C. E. Ford, J. W. Meador, R. A. Ferre, E. L. Brown, S. L. Binford, M. A. Brothers, D. M. DeLisle, and S. T. Worland. 1999. Structure- assisted design of mechanism-based irreversible inhibitors of human rhinovirus $3 \mathrm{C}$ protease with potent antiviral activity against multiple rhinovirus serotypes. Proc. Natl. Acad. Sci. USA 96:11000-11007.

29. Otwinowski, Z., and W. Minor. 1997. Processing of X-ray diffraction data collected in oscillation mode, p. 307-326. In C. W. Carter, Jr., and R. M. Sweet (ed.), Macromolecular crystallography, part A, vol. 276. Academic Press, New York, NY.

30. Pyrc, K., B. Berkhout, and L. van der Hoek. 2007. The novel human coronaviruses NL63 and HKU1. J. Virol. 81:3051-3057.

31. Sloots, T. P., P. McErlean, D. J. Speicher, K. E. Arden, M. D. Nissen, and I. M. Mackay. 2006. Evidence of human coronavirus HKU1 and human bocavirus in Australian children. J. Clin. Virol. 35:99-102.

32. Storoni, L. C., A. J. McCoy, and R. J. Read. 2004. Likelihood-enhanced fast rotation functions. Acta Crystallogr. D Biol. Crystallogr. 60:432-438.

33. Thompson, J. D., D. G. Higgins, and T. J. Gibson. 1994. CLUSTAL W: improving the sensitivity of progressive multiple sequence alignment through sequence weighting, position-specific gap penalties and weight matrix choice. Nucleic Acids Res. 22:4673-4680.

34. Vabret, A., J. Dina, S. Gouarin, J. Petitjean, S. Corbet, and F. Freymuth. 2006. Detection of the new human coronavirus HKU1: a report of 6 cases. Clin. Infect. Dis. 42:634-639.

35. van der Hoek, L., K. Pyrc, and B. Berkhout. 2006. Human coronavirus NL63, a new respiratory virus. FEMS Microbiol. Rev. 30:760-773.

36. Vijgen, L., E. Keyaerts, P. Lemey, P. Maes, K. Van Reeth, H. Nauwynck, M. Pensaert, and M. Van Ranst. 2006. Evolutionary history of the closely related group 2 coronaviruses: porcine hemagglutinating encephalomyelitis virus, bovine coronavirus, and human coronavirus OC43. J. Virol. 80:72707274.

37. Wang, L. F., Z. Shi, S. Zhang, H. Field, P. Daszak, and B. T. Eaton. 2006 Review of bats and SARS. Emerg. Infect. Dis. 12:1834-1840.

38. Woo, P. C., Y. Huang, S. K. Lau, H. W. Tsoi, and K. Y. Yuen. 2005. In silico analysis of ORF1ab in coronavirus HKU1 genome reveals a unique putative cleavage site of coronavirus HKU1 3C-like protease. Microbiol. Immunol. 49:899-908.

39. Woo, P. C., S. K. Lau, C. M. Chu, K. H. Chan, H. W. Tsoi, Y. Huang, B. H. Wong, R. W. Poon, J. J. Cai, W. K. Luk, L. L. Poon, S. S. Wong, Y. Guan, J. S. Peiris, and K. Y. Yuen. 2005. Characterization and complete genome sequence of a novel coronavirus, coronavirus HKU1, from patients with pneumonia. J. Virol. 79:884-895.

40. Wu, P. S., L. Y. Chang, B. Berkhout, L. van der Hoek, C. Y. Lu, C. L. Kao, P. I. Lee, P. L. Shao, C. Y. Lee, F. Y. Huang, and L. M. Huang. 2008. Clinica manifestations of human coronavirus NL63 infection in children in Taiwan. Eur. J. Pediatr. 167:75-80.

41. Xue, X., H. Yu, H. Yang, F. Xue, Z. Wu, W. Shen, J. Li, Z. Zhou, Y. Ding, Q. Zhao, X. C. Zhang, M. Liao, M. Bartlam, and Z. Rao. 2008. Structures of two coronavirus main proteases: implications for substrate binding and antiviral drug design. J. Virol. 82:2515-2527.

42. Yang, H., M. Bartlam, and Z. Rao. 2006. Drug design targeting the main protease, the Achilles' heel of coronaviruses. Curr. Pharm. Des. 12:45734590.

43. Yang, H., W. Xie, X. Xue, K. Yang, J. Ma, W. Liang, Q. Zhao, Z. Zhou, D. Pei, J. Ziebuhr, R. Hilgenfeld, K. Y. Yuen, L. Wong, G. Gao, S. Chen, Z. Chen, D. Ma, M. Bartlam, and Z. Rao. 2005. Design of wide-spectrum inhibitors targeting coronavirus main proteases. PLoS Biol. 3:e324.

44. Yang, H., M. Yang, Y. Ding, Y. Liu, Z. Lou, Z. Zhou, L. Sun, L. Mo, S. Ye, H. Pang, G. F. Gao, K. Anand, M. Bartlam, R. Hilgenfeld, and Z. Rao. 2003 The crystal structures of severe acute respiratory syndrome virus main protease and its complex with an inhibitor. Proc. Natl. Acad. Sci. USA 100: 13190-13195.

45. Zakstel'skaia, L., and A. V. Sheboldov. 1971. New group of viruses: corona viruses. Vestn. Akad. Med. Nauk SSSR. 26:64-69.

46. Zheng, W. X., L. L. Chen, H. Y. Ou, F. Gao, and C. T. Zhang. 2005. Coronavirus phylogeny based on a geometric approach. Mol. Phylogenet. Evol. 36:224-232.

47. Zhu, R. N., Y. Qian, L. Q. Zhao, J. Deng, F. Wang, and B. Liao. 2006. Human coronavirus-NL63 was detected in specimens from children with acute respiratory infection in Beijing, China. Zhonghua Er Ke Za Zhi 44:202-205. 